\title{
BCG Solution
}

National Cancer Institute

\section{Source}

National Cancer Institute. BCG Solution. NCI Thesaurus. Code C83496.

A solution containing an attenuated, live culture preparation of the Bacillus Calmette Guerin (BCG) strain of Mycobacterium bovis with potential immunostimulating activity. Although the precise mechanism of action is unknown, upon intravesical administration, attenuated, live BCG bacteria in the solution come into direct contact with the bladder wall, inciting an antitumor granulomatous inflammatory reaction. 